\title{
ventilación natural de túneles de ferrocarril
}

JOSE LAORDEN, Ingeniero de Caminos, Jefe del Departamenio de Instalaciones del Instituto Eduardo Torroja de la Construeción y del Cemento.

RAFAEL MUÑOZ MARTIALAY, del Departamento de Instalaciones del Instituto Eavardo Torroja de la Construcción y del Cemento.

$313-3$

\section{Sinopsis}

En este artículo se describen, de un modo resumido, los cálculos y resultados que se han obtenido al estudiar el problema de la ventilación natural de un túnel para ferrocarril de vapor, en el Norte de España, de $2.370 \mathrm{~m}$ de longitud y una pendiente de 15 milésimas.

Este estudio se realizó a petición bajo la supervisión y coordinación del Ing. de Caminos D. José Hidalgo, Director de Hidroeléctrica de Galicia. Parte de los datos experimentales fueron proporcionados por el Ingeniero de Caminos Sr. Gutiérrez, también de Hidroeléctrica de Galicia.

Los autores agradecen sinceramente al Ingeniero D. Luis Blanco, Jefe de Formación Profesional de la Renfe, los datos que les proporcionó sobre los accidentes en túnel de ferrocarril, así como sus consejos sobre la forma práetica de resolver el problema, basados en su gran experiencia del tema.

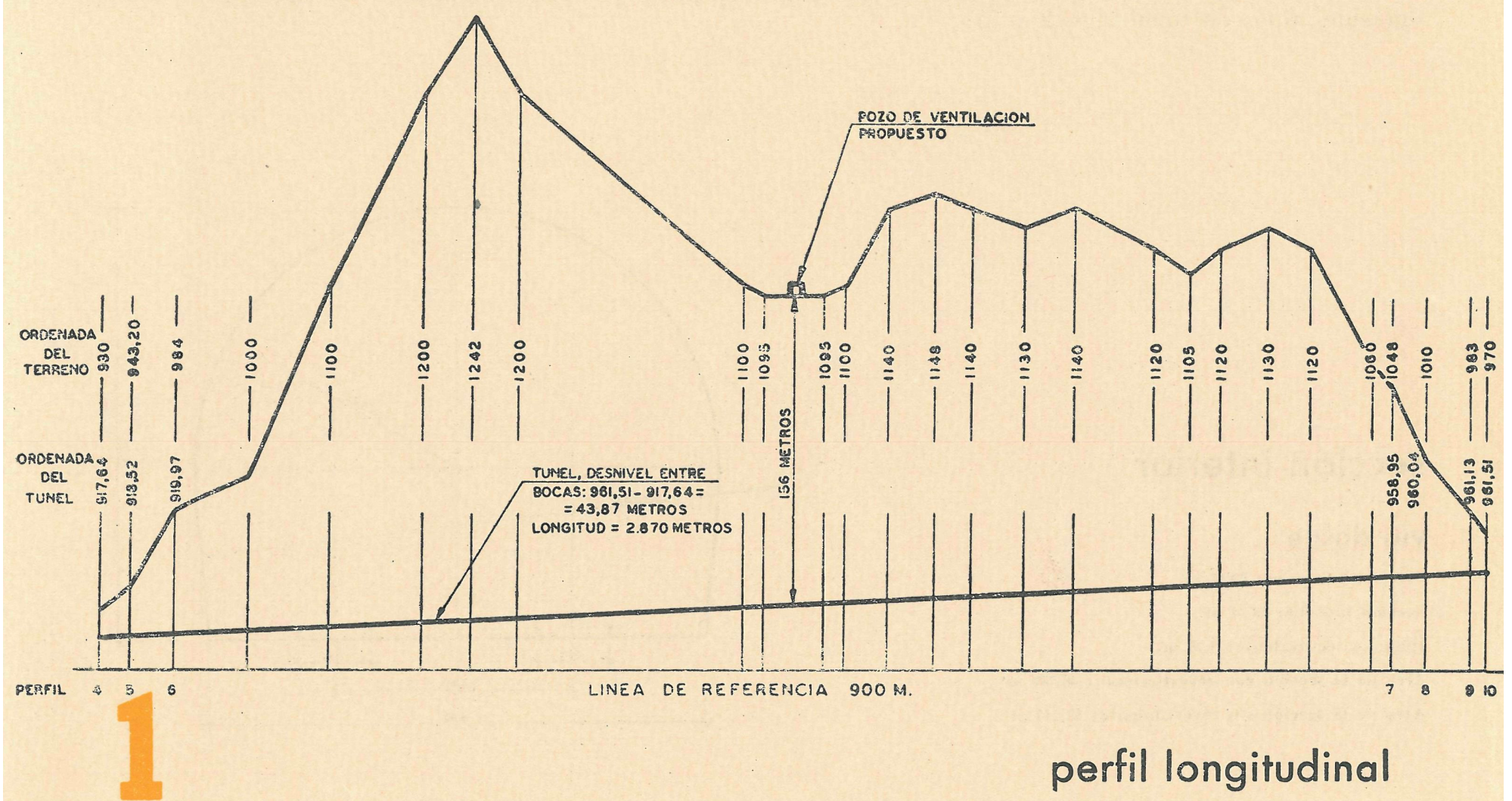




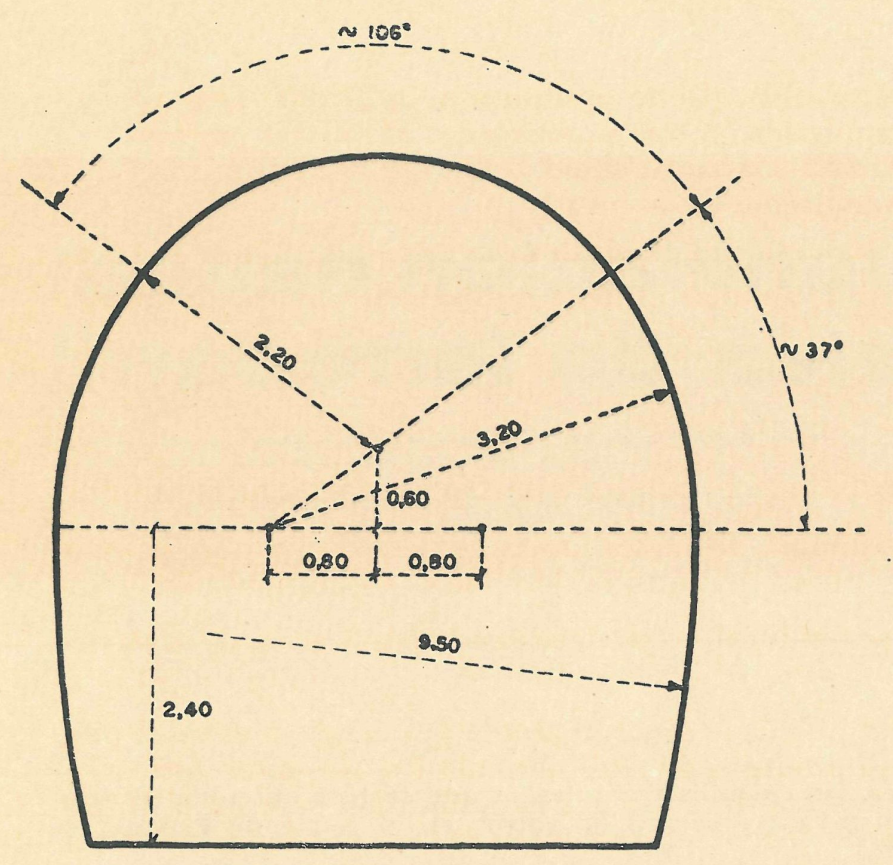

\section{sección interior}

vía única

Perímetro interior: $17,45 \mathrm{~m}$.

Sección interior:

$\frac{4,25+4,80}{2} \times 2,40+\pi \times 2,20^{2} \times \frac{106}{360}+2 \pi \times 3,20^{2} \times \frac{37}{360}-\frac{1}{2}-1,60 \times 0,60=$

$=10,88+4,48+6,62-0,48=21,50 \mathrm{~m}^{2}$.

Diámetro equivalente : $\frac{4 \Omega}{P}=\frac{4 \times 21,50}{17,45}=4,93 \mathrm{~m}$.

Area de la sección con revestimiento: $28 \mathrm{~m}^{2}$.

Area de la sección sin revestimiento: $21,50 \mathrm{~m}^{2}$.

\section{Antecedentes}

Al tener que realizar, en una zona del Norte de España, la desviación de un ferrocarril, se tuvo que proyectar la construcción del mencionado túnel, en el que, dada su longitud y pendiente, y el tipo de trenes que iban a circular por él (trenes mineros), resultaba de gran interés conocer si se podría producir la asfixia del personal del tren en el caso extremo de que las circulaciones se realizasen circunstancialmente a velocidades muy reducidas-del orden de los $17 \mathrm{~km} / \mathrm{hora}$-, tardando unos diez minutos en efectuar su total recorrido.

Los problemas de ventilación natural se han estudiado para el trazado indicado en la figura 1 y para los siguientes tipos de túnel (figs. 2 y 3 ):

\section{sección interior}

\section{vía doble}

Perímetro interior: $22,85 \mathrm{~m}$.

Sección interior: $38,24 \mathrm{~m}^{2}$.

Diámetro equivalente: $6,75 \mathrm{~m}$.

Area de la sección con revestimiento: $48,787 \mathrm{~m}^{2}$.

Area de la sección sin revestimiento: $38,244 \mathrm{~m}^{2}$.

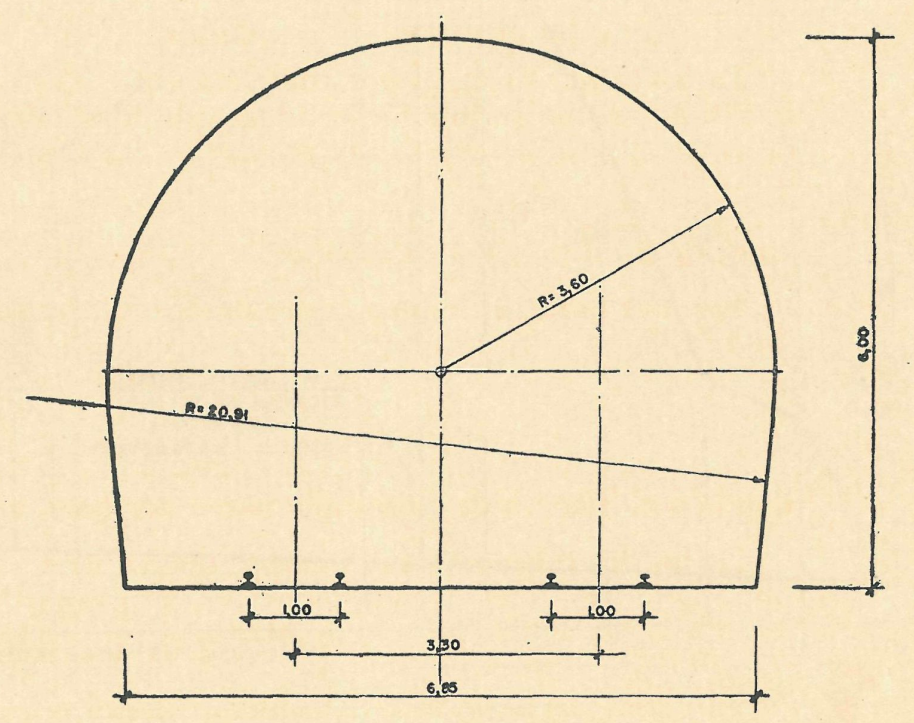


a) Túnel de vía única sin chimenea de ventilación. (Coste aproximado, 36.270.000 pesetas).

b) Túnel de vía doble sin chimenea de ventilación. (Coste aproximado, 55.860.000 pesetas).

c) Túnel de vía única con chimenea de ventilación en su mitad.

d) Túnel de vía doble con chimenea de ventilación en su mitad.

Para las chimeneas de ventilación se han supuesto los siguientes diámetros: $3 ; 3,5 ; 4$, y 4,5 m.

En este artículo no se estudia la posibilidad de ventilar el túnel mediante la circulación forzada de los humos.

\section{Planteamiento del problema}

Los dos problemas básicos que se plantean cuando se estudia la ventilación de un túnel son:

1..$^{-}$Evitar que en el túnel se vayan concentrando los gases tóxicos, producidos por las locomotoras, ya que impediría la entrada de nuevos trenes; así como las eventuales reparaciones de la vía.

2. Examinar si durante el paso del tren, por el túnel, se produce una impurificación de la atmósfera tal, que perjudique al personal del tren.

Para resolver el primer punto hay que tratar de que el túnel se ventile por conpleto entre el paso de cada dos trenes consecutivos, sin que se vaya produciendo una acumulación de gases tóxicos, y para el segundo hay que conseguir que la atmósfera no sufra una impurificación superior al 10 por mil alrededor del tren, si se trata de proteger a los fogoneros, o al 6 por mil en el interior del túnel, si se considera que han de entrar obreros para la conservación de la vía *.

La ventilación del túnel entre el paso de cada dos trenes depende, fundamentalmente, de:

a) Tipo de túnel y chimenea de ventilación.

b) Velocidad del tren.

c) Número de trenes que pasan por el túnel.

d) Diferencia de temperaturas entre el aire del túnel y el aire exterior.

La impurificación de la atmósfera alrededor del tren depende, esencialmente, de:

a) Velocidad del tren.

b) Velocidad inicial del aire circulando por el túnel antes de la entrada del tren.

Esta última variable viene ligada al tipo de túnel, a la diferencia de temperaturas entre el aire exterior y el del túnel, etc.

\section{Ventilación del túnel entre el paso de cada dos trenes consecutivos}

En el estudio que se hace a continuación se trata de obtener la mínima diferencia de temperaturas necesaria entre el aire del túnel y el aire exterior para que se produzca una corriente de aire dentro del túnel que lo ventile por completo entre el paso de cada dos trenes consecutivos:

a) Túnel sin chimenea de ventilación.

Para ventilar el túnel por completo entre el paso de cada dos trenes consecutivos será necesario mover el aire, en su interior, con una velocidad mínima, $u$, tal que, durante el tiempo libre, $t$, que quede entre el paso de dos trenes, recorra los $2.870 \mathrm{~m}$ de longitud que tiene el túnel, o sea:

$$
2.870=u \cdot t \cdot 60 \text {, }
$$

viniendo $u$ en $\mathrm{m} / \mathrm{s}$ y $t$ en minutos.

Por otra parte, el tiempo $t$, en minutos, que queda libre entre el paso de dos trenes consecutivos es:

$$
t=60-n \frac{2.870}{\frac{1.000 \mathrm{~V}}{60}}
$$

siendo $n$ el número de trenes que pasan por hora, y $\mathrm{V}$, la velocidad en $\mathrm{km} /$ hora.

De las dos relaciones anteriores se deduce que:

$$
u=\frac{1.435 \mathrm{~V}}{1.800 \mathrm{~V}-5.166 \mathrm{n}}
$$

\footnotetext{
* Estos límites son los impuestos en las Normas italianas, ya que no se disponían de datos de los ferrocarriles españoles.
} 
Ahora bien, cuando el aire se mueve en el túnel con la velocidad $u$ se produce un razomiento, $e$, que viene dado, en mm de columna de agua, por la expresión:

$$
e=\frac{u^{2}}{2 \mathrm{~g}}+\rho \frac{\mathrm{L}}{\mathrm{D}} \cdot \frac{u^{2}}{2 \mathrm{~g}}
$$

en la cual D es el diámetro equivalente del túnel, en metros; g, la aceleración de la gravedad, en m/ $\mathrm{s}^{2}$; $L$, la longitud del túnel en metros, y $\rho$, el coeficiente de rozamiento unitario del aire en el túnel.

El primer sumando corresponde a la presión debida a la velocidad, y el segundo sumando, al rozamiento a lo largo del túnel.

La expresión [III] aplicada a nuestro caso se reduce a:

$$
e=0,054\left(1+\rho \frac{2.870}{\mathrm{D}}\right) u^{2}
$$

Considerando una presión atmosférica de $680 \mathrm{~mm}$, y despreciando la influencia de la humedad relativa (esta influencia, para temperaturas del aire entre 0 y $40^{\circ} \mathrm{C}$, no excede del $4 \%$, según el ábaco del «Manual Sulzer») se obtiene, de un modo muy aproximado, el valor de la presión $e$ :

$$
e=0,16 \mathrm{~T} \text {, }
$$

siendo $\mathrm{T}$ la diferencia de temperaturas, en grados centígrados, entre el aire exterior y el interior.

Sustituyendo este valor de $e$ en [IV] se llega a:

$$
\mathrm{T}=0,3375 u^{2}\left(1+2.870 \frac{\rho}{\mathrm{D}}\right)
$$

Finalmente, si sustituímos el valor de $u$ deducido en [II], nos queda:

$$
\mathrm{T}=695.000\left(1+2.870 \frac{\rho}{\mathrm{D}}\right) \frac{\mathrm{V}^{2}}{(1.800 \mathrm{~V}-5.166 n)^{2}}
$$

Esta fórmula aplicada a los casos de:

$$
\text { Túnel de vía única } \mathrm{D}=4,93 \mathrm{~m} \begin{cases}\text { Superficie revestida: } & \rho=0,02 \\ \text { Superficie rugosa: } & \rho=0,04\end{cases}
$$

nos da los siguientes valores:

$$
\left.\begin{array}{ll}
\text { Vía única, rugosa: } & \mathrm{T}=16.900 .000 \\
\text { Vía única, revestida: } & \mathrm{T}=8.800 .000 \\
\text { Vía doble, rugosa: } & \mathrm{T}=12.500 .000 \\
\text { Vía doble, revestida: } & \mathrm{T}=6.600 .000
\end{array}\right\} \times \frac{\mathrm{V}^{2}}{(1.800 \mathrm{~V}-5.166 n)^{2}}
$$

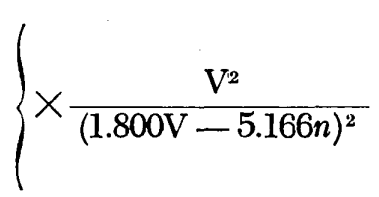

b) Túnel con chimenea de ventilación.

El razonamiento es análogo al caso anterior, pero hay que suponer que el aire recorre sólo la mitad de la longitud del túnel, pues la chimenea se debe colocar en el centro del mismo.

Aplicando ahora el coeficiente de rozamiento correspondiente, y que también es distinto del anterior, se llega a la siguiente expresión:

$$
\mathrm{T}=\frac{57.000 \mathrm{~V}^{2}}{(1.800 \mathrm{~V}-5.166 n)^{2}}\left[4 \frac{\mathrm{S}^{2}}{\mathrm{Q}^{2}}\left(1+0,02 \frac{\lambda}{\delta}\right)+\rho \frac{2.870}{2 \mathrm{D}}\right]
$$

siendo:

$\mathrm{T}=$ diferencia de temperaturas entre el aire interior del túnel y el exterior, en ${ }^{\circ} \mathbf{C}$.

$\rho=$ coeficiente de rozamiento del aire en el túnel.

$\mathrm{D}=$ diámetro equivalente del túnel.

$\mathrm{V}=$ velocidad del tren, en $\mathrm{km} / \mathrm{h}$. 


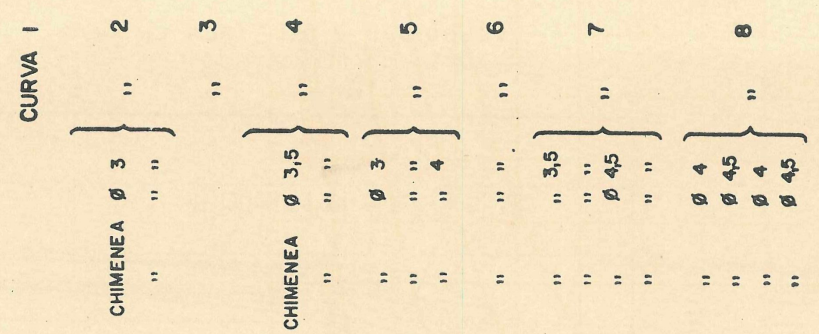

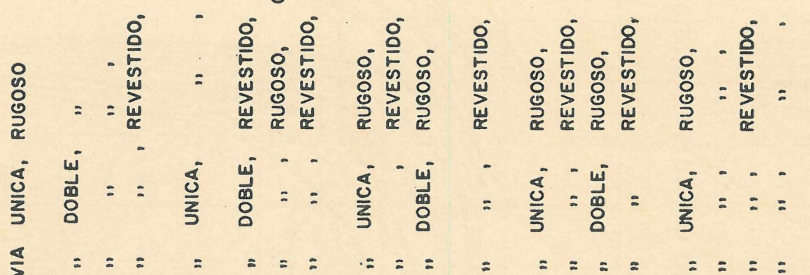
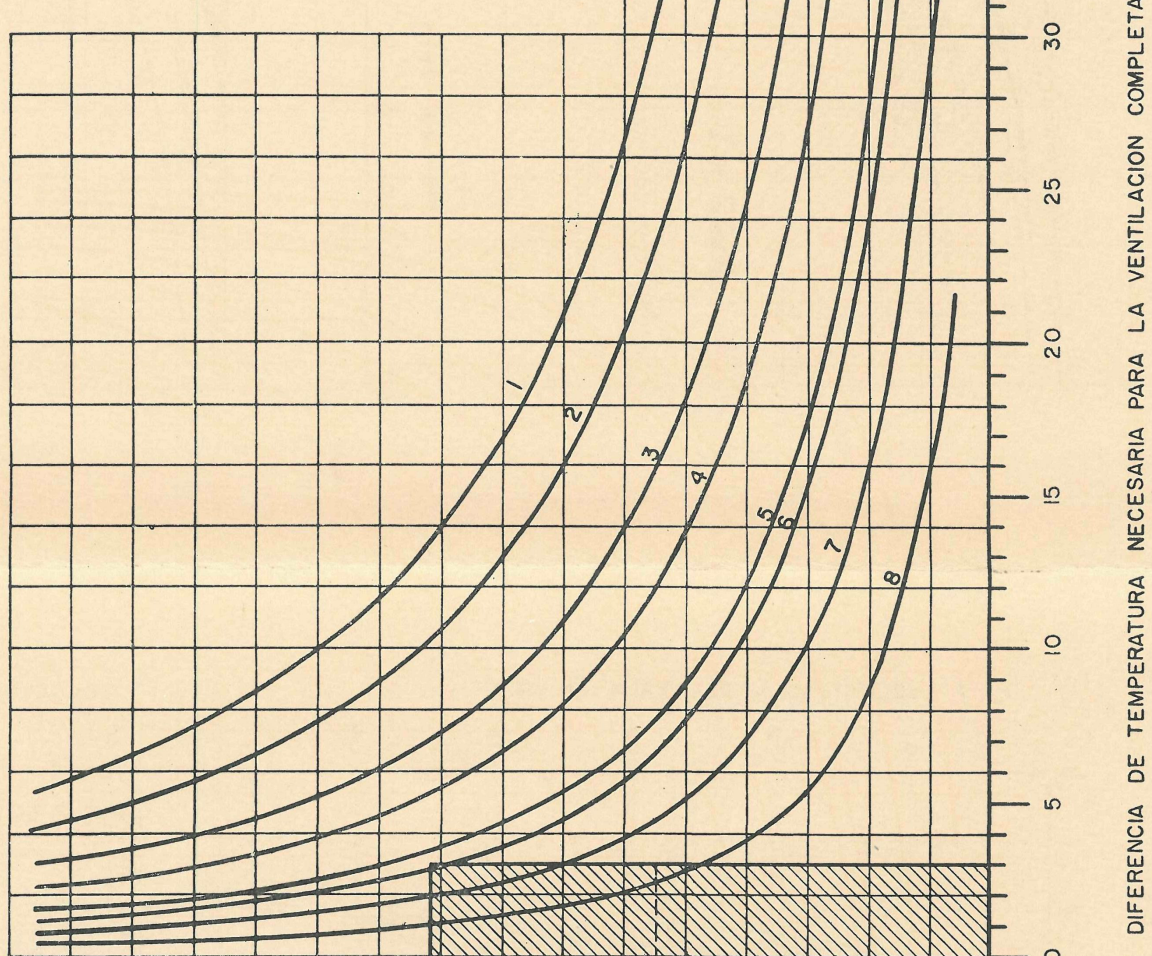

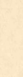

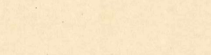



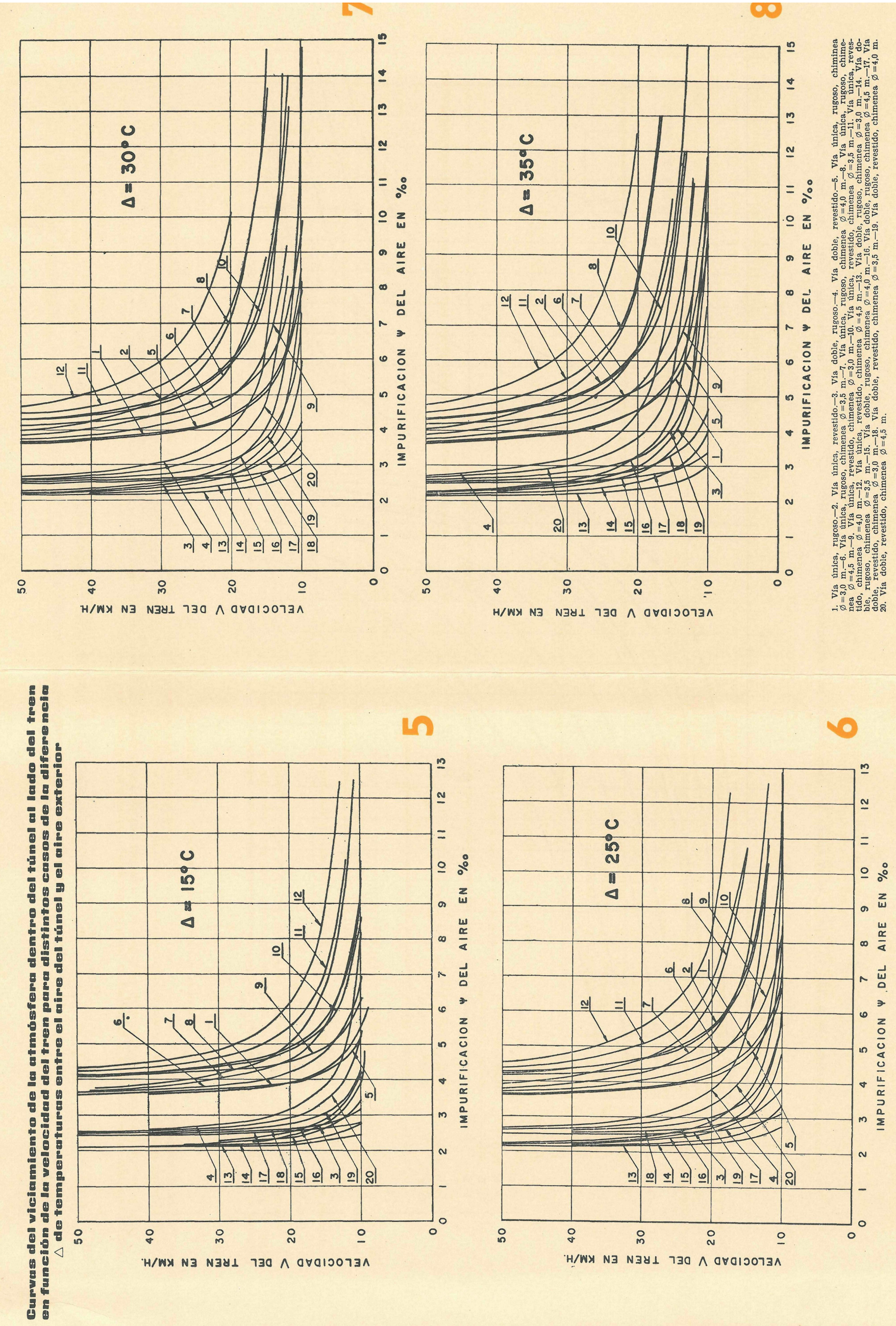
b) El aire se mueve en la misma dirección que el tren:

$$
\psi_{2}=\frac{C}{S \cdot L\left(1-\frac{u}{V}\right)}
$$

Siendo:

$\mathrm{C}=$ cantidad total de $\mathrm{CO}_{2}+\mathrm{C}_{0}$ producida $=78 \mathrm{~m}^{3}$.

$\mathrm{S}=$ sección del túnel en $\mathrm{m}^{2}$.

$\mathrm{L}=$ longitud del túnel $=2.870 \mathrm{~m}$.

$u=$ velocidad del aire, en el túnel, en $\mathrm{m} /$ segundo.

$\mathrm{V}=$ velocidad del tren en $\mathrm{m} /$ segundo.

De la expresión [V] se deduce que:

$$
u=1,72 \sqrt{\frac{T}{1+2.870 \frac{p}{D}}}
$$

Sustituyendo este valor de $u$ en las fórmulas [VI] y [VII], queda finalmente:

$$
\begin{aligned}
& \left.\psi_{1}=\frac{\mathrm{C}}{\mathrm{S} \cdot \mathrm{L}\left[1,72 \sqrt{\frac{\mathrm{T}}{1+2.870 \frac{\rho}{\mathrm{D}}}}\right.}\right] \\
& \left.\psi_{2}=\frac{\mathrm{C}}{\mathrm{S} \cdot \mathrm{L}\left[1-\frac{1,72 \sqrt{\frac{\mathrm{T}}{1+2.870 \frac{\rho}{\mathrm{D}}}}}{\mathrm{V}}\right.}\right]
\end{aligned}
$$

Estas expresiones se han representado para distintos valores de $T$ en los gráficos de las figuras 5 , 6,7 y 8 , indicándose la impurificación del aire en tanto por mil y reduciendo al $70 \%$ el grado de impurificación, ya que los gases suelen acumularse en el techo.

\section{Conclusiones}

A la vista de los gráficos de las figuras $3,5,6,7$ y 8 , se deduce que:

1. Atendiendo a la ventilación natural del túnel y a la frecuencia de trenes (dos a la hora), el túnel, sea de vía única o de vía doble, necesita tener una chimenea de ventilación o una ventilación forzada.

2. Atendiendo al viciamiento de la atmósfera, alrededor del tren, para trenes que marchen con velocidad mínima de $17 \mathrm{~km} / \mathrm{h}$ se obtiene que el viciamiento dentro de un túnel de vía única y chimenea de $3,5 \mathrm{~m}$ de diámetro será inferior al 6 por mil.

3. ${ }^{\circ}$ Si el tren marchase por el túnel con una velocidad de $10 \mathrm{~km} / \mathrm{h}$, en el caso de túnel de vía única y chimenea de $3 \mathrm{~m}$ de diámetro el viciamiento sería del 8,23 por mil para una diferencia de temperaturas de $25^{\circ} \mathrm{C}$.

4. Un túnel de vía única con una chimenea de $3,5 \mathrm{~m}$ de diámetro tendría asegurada la ventilación natural del túnel entre cada dos trenes consecutivos, siempre que la diferencia de temperaturas entre el aire exterior y el del túnel fuese, como mínimo, de $3^{\circ} \mathrm{C}$, la frecuencia de trenes no fuese superior a dos a la hora y la velocidad del tren fuese, como mínimo, de $12 \mathrm{~km} / \mathrm{h}$. En este mismo túnel el viciamiento de la atmósfera alrededor del tren no excedería del 9,17 por mil, siempre que marchase, por lo menos, a $12 \mathrm{~km} / \mathrm{h}$, la diferencia de temperatura no excediese de $30^{\circ} \mathrm{C}$ y el túnel fuese rugoso. 